\title{
Improved Quality of Service for Video, Data and Voice Integrated Wireless Cellular Networks through the Queue Dynamic Channel Reservation Scheme
}

\author{
Chinedu Duru \\ Department of Electronic Engineering \\ University of Nigeria, Nsukka
}

\author{
Mamilus Ahaneku \\ Department of Electronic Engineering \\ University of Nigeria, Nsukka
}

\begin{abstract}
Most cellular wireless networks are voice, data, and video integrated which now introduces the challenge on providing channel allocation techniques to ensure adequate performance for all services. Furthermore, with the increasing demand for these mobile multimedia services, cellular networks have adopted micro architectures to provide the higher capacity needed to support the broadband high speed of these services under the limited radio spectrum. This consequently has increased the undesirable interruption of ongoing calls as the mobile terminal moves from one cell to another. Handoff calls, as they are called, need to perform well and introducing a scheme that gives priority to handoff calls over new calls is necessary. This paper proposes a scheme that guarantees the Quality of Service (QoS) of handoff calls and new calls. The proposed design, based on the combining features of the existing Dynamic Channel Reservation Scheme (DCRS) and the Handoff Queuing Scheme (HQS), ensures both a low dropping probability of handoff calls and a low blocking probability of new calls with an improved channel utilization without system overload. It would be shown that through the analytical results of the proposed design, namely the Queue Dynamic Channel Reservation Scheme (QDCRS), compared with the Fully Shared Channel Scheme (FSS); offers exceptional QoS with the opportunity for added improvement performance.
\end{abstract}

\section{General Terms}

Wireless Cellular Networks, dynamic channel allocation, multimedia services.

\section{Keywords}

Guard channel; allocation; queue; dynamic; handoff; Quality of Service (QoS); Wireless Cellular Networks; multimedia services

\section{INTRODUCTION}

In cellular wireless networks, channel allocation methods are important to ensure a fair balance between the user and the service provider. From the user's perspective, what is desired is a better performance in handoff dropping calls than new calls; whereas from the service provider's point of view, a system that can efficiently maximise utilisation without overload is preferred. Nowadays, most cellular wireless networks are voice, data, and video integrated which now introduces the challenge on providing channel allocation techniques to ensure adequate performance and satisfaction for all services [1]. Furthermore, with the increasing demand for these mobile multimedia services, cellular networks have adopted micro architectures to provide the higher capacity needed to support the broadband high speed of these services under the limited radio spectrum. The service provider solution has been to make the cell sizes smaller for regions [2][3]. This consequently has made handoff calls to occur frequently primarily because the small cell sizes find it difficult to accommodate the higher capacity on the limited radio spectrum as a result of no idle channels at the base station (BS). Importantly, as the handoff rate increases, bandwidth management, call admission control, and handoff procedure become a problem in the cellular network [4]. Since the network system is designed to suit users' satisfaction and because a mobile user prefers the dropping of a new call than the dropping of a progressing call as the mobile terminal moves from one cell to another, increasing handoff call performance is the ultimate goal [5][6][7].

Handoff prioritization can be classified into two methods, namely the guard channel method and the queue method. The guard channel method is a technique where a small portion of a high system channel capacity limit is exclusively reserved for handoff requests whereas the remaining normal channels are shared between handoff and new calls. This consequently gives higher priority to handoff calls and helps to reduce forced termination [8]. These guard channels can either be fixed or dynamically allocated. For fixed allocation, the guard channels are developed under the assumption of stationary call arrivals which may not work well in terms of performance and quality of service (QoS) when the total carried traffic arrive under non-stationary traffic patterns due to fluctuation in mobility. For dynamic channel allocation, on the other hand, the characteristics of traffic mobility are taken into account and channels are allocated dynamically based on those characteristics [9]. This helps in using the limited wireless channel efficiently while satisfying the requested QoS of handoff calls.

Alternatively, handoff priority can also be provided by using the queue method for requests where a queuing system or a buffer is used for handoff calls when all channels are busy. There is no similar queue system for new calls. This increases the level of priority given to handoff traffic and minimizes loss of traffic [3]. The queue method is more suited to non real-time traffic (i.e. data and video) and less suited to realtime traffic (i.e. voice) where delays, brought upon by the queue process until a channel is available, cannot be tolerated.

This paper proposes a scheme that guarantees the QoS, that is the new call blocking probability $\left(\mathrm{P}_{\mathrm{b}}\right)$, the handoff call dropping probability $\left(\mathrm{P}_{\mathrm{d}}\right)$ and the system utilization $(\mathrm{u})$, for a voice/data/video integrated cellular wireless network. The proposed scheme is based on the combination of the Dynamic Channel Reservation Scheme (DCRS) proposed by Source [9] and the Handoff Queuing Scheme (HQS) [10][11][12]. The DCRS is where the guard channels reserved for handoff calls are also used to accommodate new 
calls. The dynamic allocation of new calls to the guard channels is determined by an acceptance probability that depends on the mobility of calls, the total number of available channels, the threshold between normal and guard channels, and the current number of occupied channels in the cell. The HQS on the other hand, is where a queue system is dedicated to handoff calls while there is no similar system for new calls. It would be discovered that with the proposed scheme, namely the Queue Dynamic Channel Reservation Scheme (QDCRS), compared with the Fully Shared Channel Scheme (FSS), will guarantee both a low dropping probability of handoff calls and a low blocking probability of new calls with an improved channel utilization without system overload. Additionally, it would also be shown that the QDCRS can give room for even more additional performance improvement of QoS than the FSS for the integrated network of voice, data, and video.

\section{SYSTEM MODEL}

Consider the model of Figure 1. Here, the three classes of traffic, namely voice (real-time), data (non-real time) and video (non-real time) arrive at a base station (BS). The traffic is classified as new and handoff determined by the type of request. It is assumed that all cells are statistically identical and therefore a single cell is focused on. It is also assumed that each admitted call of the following type; handoff video call, handoff data call, handoff voice call, new video call, new data call, and new voice call, require one channel. The total number of channels in a cell is ' $\mathrm{C}$ ' divided into five regions; T1 is the boundary between new video calls $\left(\lambda_{\text {nvid }}\right)$ and new data calls $\left(\lambda_{\text {nd }}\right)$, T2 is the boundary between new data calls $\left(\lambda_{\text {nd }}\right)$ and new voice calls $\left(\lambda_{\text {nv }}\right)$, T3 is the boundary between new voice calls $\left(\lambda_{\text {nv }}\right)$ and handoff data and handoff video calls $\left(\lambda_{\text {hd }}+\lambda_{\text {hvid }}\right)$, and T4 is the boundary between handoff data and handoff video calls $\left(\lambda_{\text {hd }}+\lambda_{\text {hvid }}\right)$ and handoff voice calls $\left(\lambda_{\mathrm{hv}}\right)$.The reason for this particular arrangement is that it gives handoff calls a distinct priority and advantage over new calls on the number of channels it can occupy. Additionally, it is assumed that users will be more engaged with a voice call than a data call, and similarly more engaged with data call than a video call.

The reservation thresholds are dynamically adjusted according to the mobility of calls. There is a queue (Q) with capacity $\mathrm{K}$ for handoff data and handoff video calls $\left(\lambda_{\text {hd }}+\lambda_{\text {hvid }}\right)$, and no similar queue for new voice calls, new video calls, new data calls, and handoff voice calls. This approach satisfies the HQS where the queue system is more supportive for non real-time traffic of which delays cannot be tolerated [11].

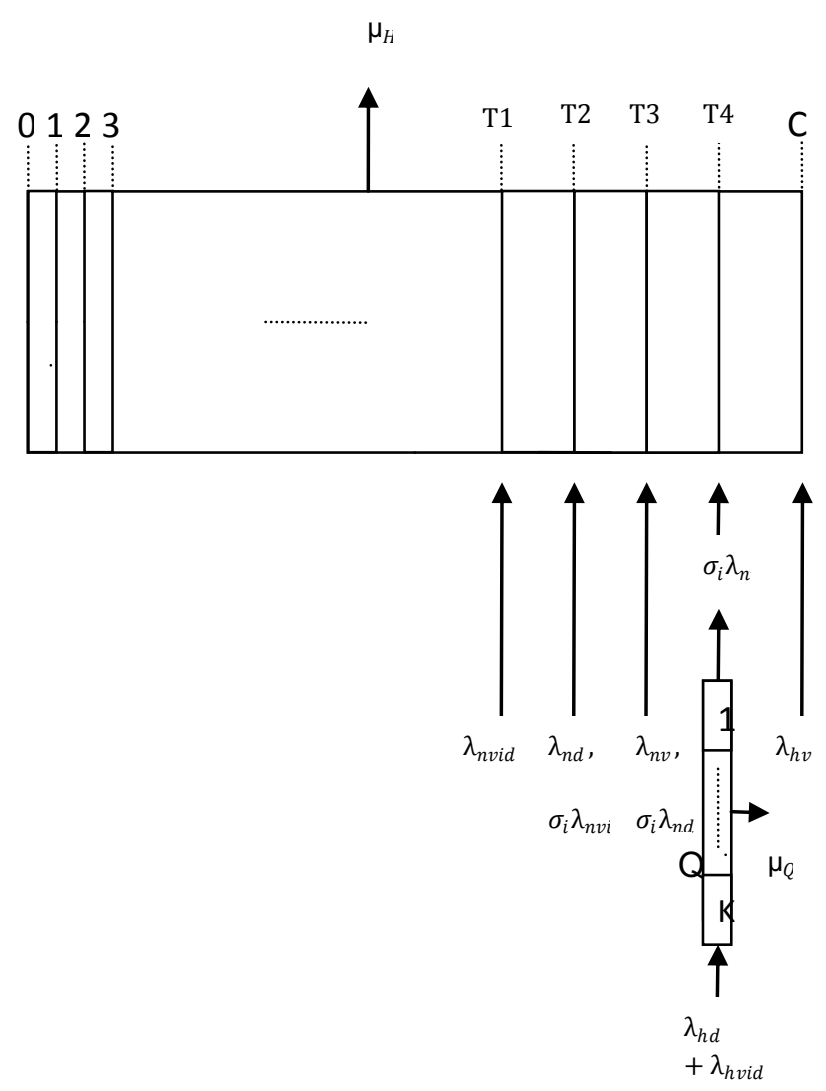

Fig 1: System Model

\subsection{Video Call Control}

New video calls arriving at the BS are accepted if the total number of occupied channels is less than T1, else they will be accepted as much as much as the acceptance probability $\sigma_{i}$ given by equation (1) [9] if the total number of occupied channels is less than T2; otherwise they are blocked.

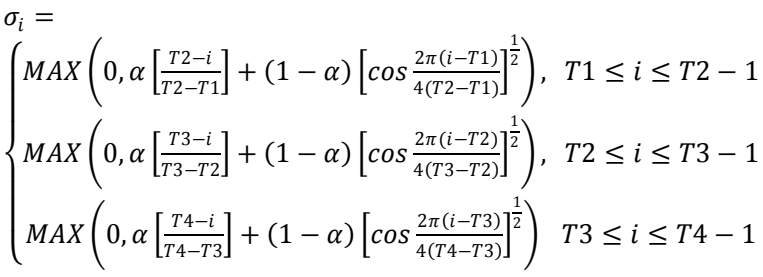

$\sigma_{i}$ is the probability a new call is accepted into the guard channels reserved for handoff calls. This is determined by the current number of occupied channels (i), the mobility of calls $(\alpha)$, and the respectable boundaries between handoff calls and new calls.

Arriving handoff video calls, on the other hand, are admitted if the total number of busy channels is less than T4. If the handoff call finds no available channel, it will be queued for a maximum $\mathrm{T}_{\mathrm{Q}}\left(=\frac{1}{\mu_{Q}}\right)$ which is the dwell time the mobile station (MS) is in the handoff area of which $\mu_{\mathrm{Q}}$ is the departure (service rate) from the queue. If a channel becomes available within $\mathrm{T} 4$ it will be assigned to a queued handoff video call on the first come first served (FCFS) basis. A handoff video call is dropped if it finds no available channel and the queue is full. It is also dropped if the call leaves the 
queue before getting a channel known as forced termination. Figure 2 demonstrates the video call admission control flow diagram.

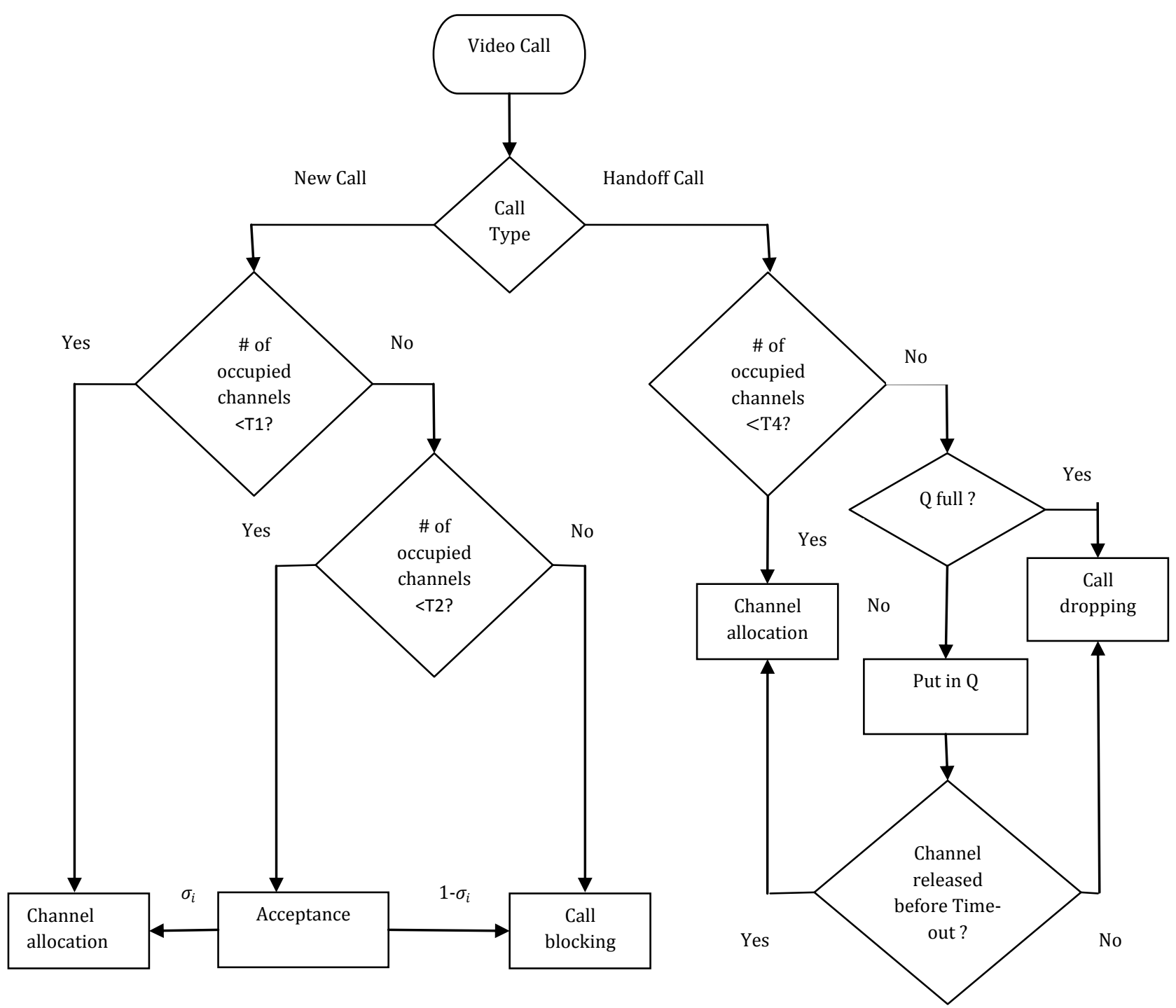

Fig 2: Video Call Admission Control Flow

\subsection{Data Call Control}

New Data calls arriving at the BS are accepted if the total number of occupied channels is less than T2 else they will be accepted as much as $\sigma_{i}$ if the total number of occupied channels is less than T3; otherwise they are blocked. On the other hand, arriving handoff data calls are admitted if the total number of busy channels is less than T4 where the same queue process that is applied to the handoff video call is also applied to the handoff data call. Figure 3 shows the data call admission control flow diagram.

\subsection{Voice Call Control}

A new voice call is accepted with probability one and assigned a channel if on arrival less than $\mathrm{T} 3$ channels are occupied; otherwise if all channels are occupied, it will enter the region reserved for handoff data calls (T4-T3) with probability $\sigma_{i}$. A new voice call will be blocked if it finds (C-
T4) channels occupied. Alternatively, when a handoff voice call arrives it will be accepted and assigned a channel if it finds a channel available; otherwise it will be dropped and cleared out. Figure 4 shows the voice call admission control flow diagram.

\section{ANALYTICAL MODEL}

The arriving calls at the BS are assumed to be generated according to a Poisson distribution process with mean arrival rates per cell $\lambda_{\text {vid }}\left(=\lambda_{\text {nvid }}+\lambda_{\text {hvid }}\right), \lambda_{\mathrm{d}}\left(=\lambda_{\text {nd }}+\lambda_{\text {hd }}\right)$, and $\lambda_{\mathrm{v}}\left(=\lambda_{\text {nv }}+\right.$ $\lambda_{\text {hv }}$ ). The total mean arrival rate is defined as [13][14]:

$$
\lambda_{\text {total }}=\lambda_{\text {vid }}+\lambda_{\mathrm{d}}+\lambda_{\mathrm{v}}
$$

The channel holding time is approximated to have an exponential distribution with mean $\mathrm{T}_{\mathrm{H}}\left(=\frac{1}{\mu_{H}}\right)$ where $\mu_{H}$ is the departure rate from the system. The normalized offered load of the system is defined as: 


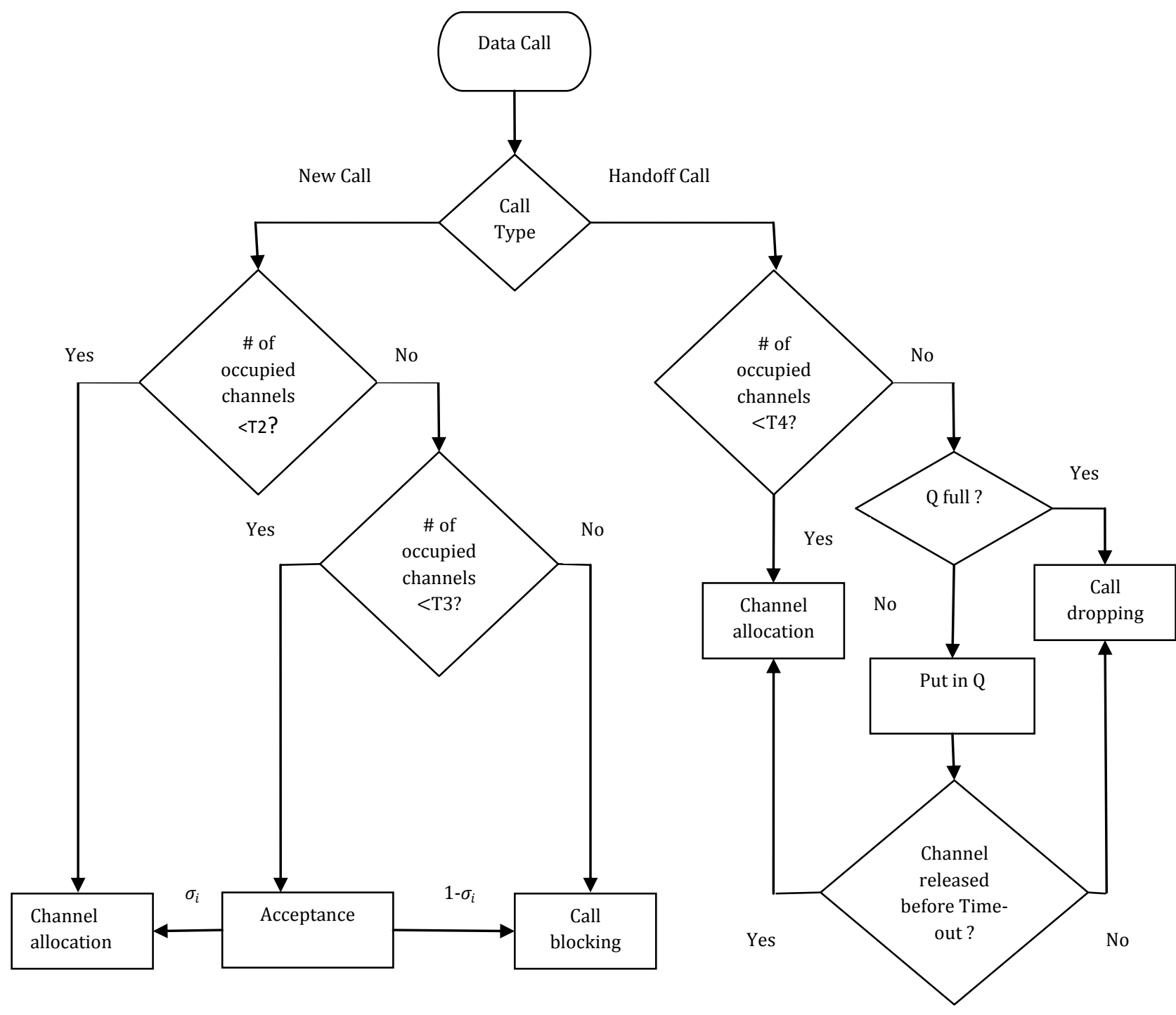

Fig 3: Data Call Admission Control Flow

$$
\rho=\frac{\lambda_{\text {total }}}{C \mu_{H}}
$$

The mobility of calls $(\alpha)$ is defined as the ratio of the mean handoff arrival rate to the mean new arrival rate, and hence the mobility of voice calls $\left(\alpha_{1}\right)$, the mobility of data calls $\left(\alpha_{2}\right)$, and the mobility of video calls $\left(\alpha_{3}\right)$ are given as [11]:

$$
\begin{gathered}
\alpha_{1}=\frac{\lambda_{h v}}{\lambda_{n v}} \\
\alpha_{2}=\frac{\lambda_{h d}}{\lambda_{n d}} \\
\alpha_{3}=\frac{\lambda_{h v i d}}{\lambda_{n v i d}}
\end{gathered}
$$

For simplicity, it is assumed that the mobility of all services are equal (i.e. $\alpha_{1}=\alpha_{2}=\alpha_{3}=\alpha$ ). Consequently, from equations (2) to (6), the mean arrival rates $\left(\lambda_{\text {hvid }}, \lambda_{\text {hd }}, \lambda_{\text {hv }}, \lambda_{\text {nvid }}\right.$, $\lambda_{\text {nd }}$, and $\lambda_{\text {nv }}$ ) can be found given the normalized offered load of the system and the mobility of calls.

\subsection{System State Probabilities}

The state of the system is defined by the two-tuple $(i, j)$ where $\mathrm{i}(\mathrm{i}=0,1,2 \ldots, \mathrm{C})$ is the number of occupied channels and $\mathrm{j}$ $(j=0,1,2 \ldots, K)$ is the number of handoff data calls waiting in the queue $(\mathrm{Q})$. Therefore the proposed scheme of the QDCRS can be modelled by a two dimensional Markov Chain as shown in Figure 5 and 6 where the balanced equations are the following:

$$
\left\{\begin{array}{c}
\left(\lambda_{\text {vid }}+\lambda_{\mathrm{d}}+\lambda_{\mathrm{v}}\right) \mathrm{P}(\mathrm{i}-1, \mathrm{j})=\mathrm{i}\left(\mu_{\mathrm{H}}\right) \mathrm{P}(\mathrm{i}, \mathrm{j}) \\
\left(\sigma_{\mathrm{i}} \lambda_{\text {nvid }}+\lambda_{\text {hvid }}+\lambda_{\mathrm{d}}+\lambda_{\mathrm{v}}\right) \mathrm{P}(\mathrm{i}-1, \mathrm{j})=\mathrm{i}\left(\mu_{\mathrm{H}}\right) \mathrm{P}(\mathrm{i}, \mathrm{j}) \\
\left(\sigma_{\mathrm{i}} \lambda_{\text {nd }}+\lambda_{\text {hd }}+\lambda_{\text {hvid }}+\lambda_{\mathrm{v}}\right) \mathrm{P}(\mathrm{i}-1, \mathrm{j})=\mathrm{i}\left(\mu_{\mathrm{H}}\right) \mathrm{P}(\mathrm{i}, \mathrm{j}) \\
\left(\sigma_{\mathrm{i}} \lambda_{\text {nv }}+\lambda_{\text {hv }}+\lambda_{\text {hd }}+\lambda_{\text {hvid }}\right) \mathrm{P}(\mathrm{i}-1, \mathrm{j})=\mathrm{i}\left(\mu_{\mathrm{H}}\right) \mathrm{P}(\mathrm{i}, \mathrm{j}) \quad(7) \\
\left(\lambda_{\text {hv }}\right) \mathrm{P}(\mathrm{i}-1, \mathrm{j})=\mathrm{i}\left(\mu_{\mathrm{H}}\right) \mathrm{P}(\mathrm{i}, \mathrm{j}) \\
\left(\lambda_{\text {hd }}+\lambda_{\text {hvid }}\right) \mathrm{P}(\mathrm{i}, \mathrm{j}-1)=\left(\mathrm{i} \mu_{\mathrm{H}}+\mathrm{j} \mu_{\mathrm{Q}}\right) \mathrm{P}(\mathrm{i}, \mathrm{j}) \\
\left(\lambda_{\text {hd }}+\lambda_{\text {hvid }}\right) \mathrm{P}(\mathrm{i}, \mathrm{j}-1)=\left(\mathrm{j} \mu_{\mathrm{Q}}\right) \mathrm{P}(\mathrm{i}, \mathrm{j})
\end{array}\right.
$$

The corresponding intervals for each balanced equation are the following: 


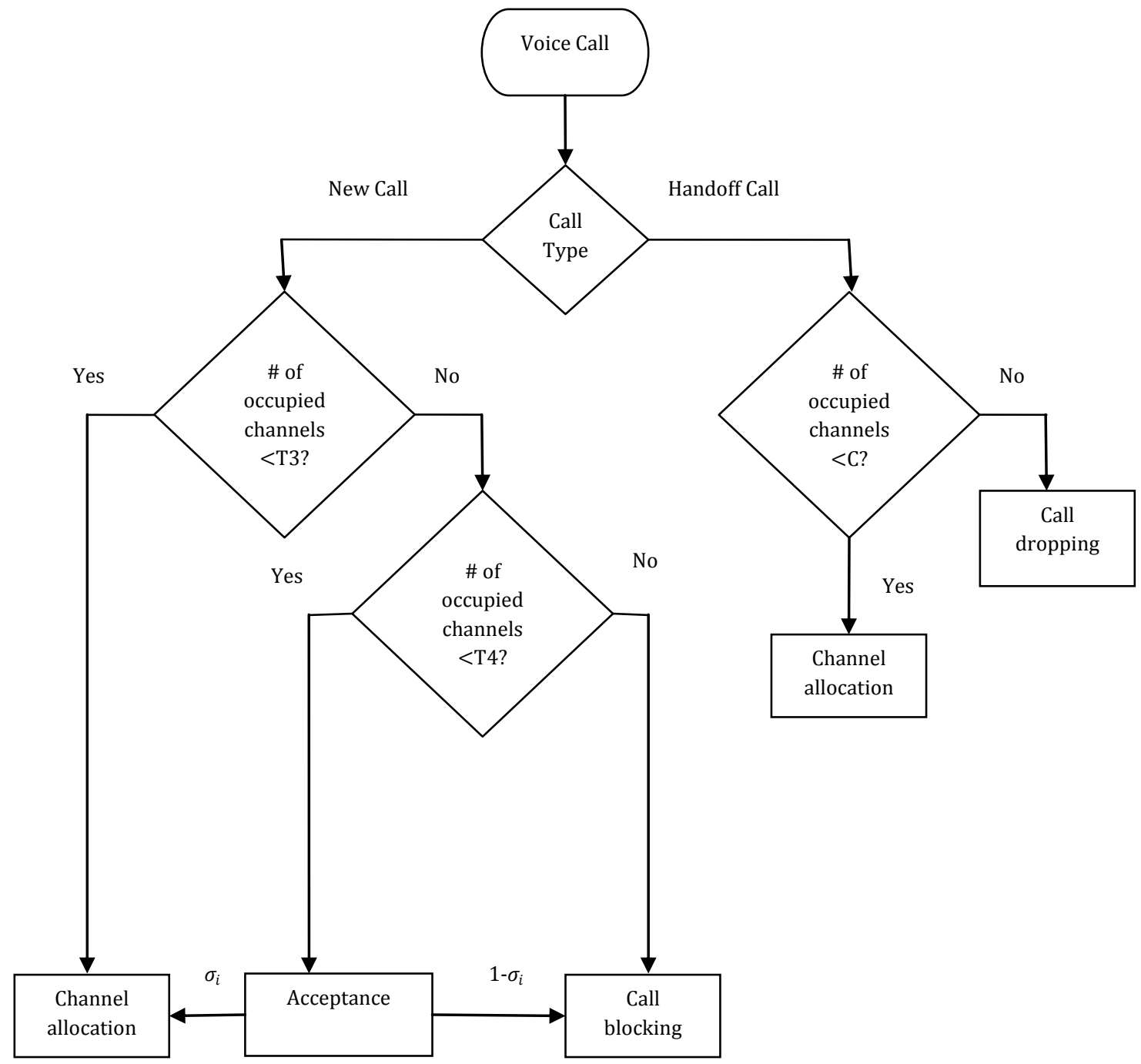

Fig 4: Voice Call Admission Control Flow

$$
\left\{\begin{array}{c}
0 \leq i \leq T 1, \mathrm{j}=0 \\
T 1+1 \leq i \leq T 2, \mathrm{j}=0 \\
T 2+1 \leq i \leq T 3, \mathrm{j}=0 \\
T 3+1 \leq i \leq T 4, \mathrm{j}=0 \\
T 4+1 \leq i \leq C, \mathrm{j}=0 \\
i=T 4,1 \leq \mathrm{j} \leq K \\
T 4+1 \leq i \leq C \quad 1 \leq \mathrm{j} \leq K
\end{array}\right.
$$

The sum of all state probabilities must equal to 1 as equation (9) shows:

$$
\sum_{i=0}^{T 4-1} P(i, 0)+\sum_{i=T 4}^{C} \sum_{j=0}^{K} P(i, j)=1
$$

Consequently, with equations (7) and (9) at intervals (8), the steady state probabilities of the system are determined as the following:

$$
=\left\{\begin{array}{c}
\frac{\left(\lambda_{\text {vid }}+\lambda_{\mathrm{d}}+\lambda_{\mathrm{v}}\right)^{i}}{i !\left(\mu_{\mathrm{H}}\right)^{i}} P(0,0) \\
\frac{\left(\lambda_{\text {vid }}+\lambda_{\mathrm{d}}+\lambda_{\mathrm{v}}\right)^{T 1} \prod_{n=1}^{i-T 1}\left(\sigma_{\mathrm{i}} \lambda_{\mathrm{nvid}}+\lambda_{\mathrm{hvid}}+\lambda_{\mathrm{d}}+\lambda_{\mathrm{v}}\right)}{i !\left(\mu_{\mathrm{H}}\right)^{i}} P(0,0) \\
\frac{\left.\left(\lambda_{\mathrm{v}}\right)^{T 1}+\lambda_{\mathrm{d}}+\lambda_{\mathrm{v}}\right)^{T 1} m 1 \prod_{n=1}^{i-T 2}\left(\sigma_{\mathrm{i}} \lambda_{\mathrm{nd}}+\lambda_{\mathrm{hd}}+\lambda_{\mathrm{hvid}}+\lambda_{\mathrm{v}}\right)}{i !\left(\mu_{\mathrm{H}}\right)^{i}} P(0,0) \\
i !\left(\mu_{\mathrm{H}}\right)^{i}\left(\sigma_{\mathrm{i}} \lambda_{\mathrm{hv}}+\lambda_{\mathrm{hv}}+\lambda_{\mathrm{hd}}+\lambda_{\mathrm{hvid}}\right) \\
\frac{\left(\lambda_{\text {vid }}+\lambda_{\mathrm{d}}+\lambda_{\mathrm{v}}\right)^{T 1} m 1 m 2 m 3 \lambda_{\mathrm{hv}}{ }^{(i-T 4)}}{i !\left(\mu_{\mathrm{H}}\right)^{i}} P(0,0) \\
\frac{\left(\lambda_{\mathrm{hd}}+\lambda_{\mathrm{hvid}}\right)^{j}}{\prod_{n=1}^{j}\left(i \mu_{\mathrm{H}}+\mathrm{n} \mu_{\mathrm{Q}}\right)} P(i, 0) \\
\frac{\left(\lambda_{\mathrm{hd}}+\lambda_{\mathrm{hvid}}\right)^{j}}{\prod_{n=1}^{j}\left(\mathrm{n} \mu_{\mathrm{Q}}\right)} P(i, 0)
\end{array}\right.
$$




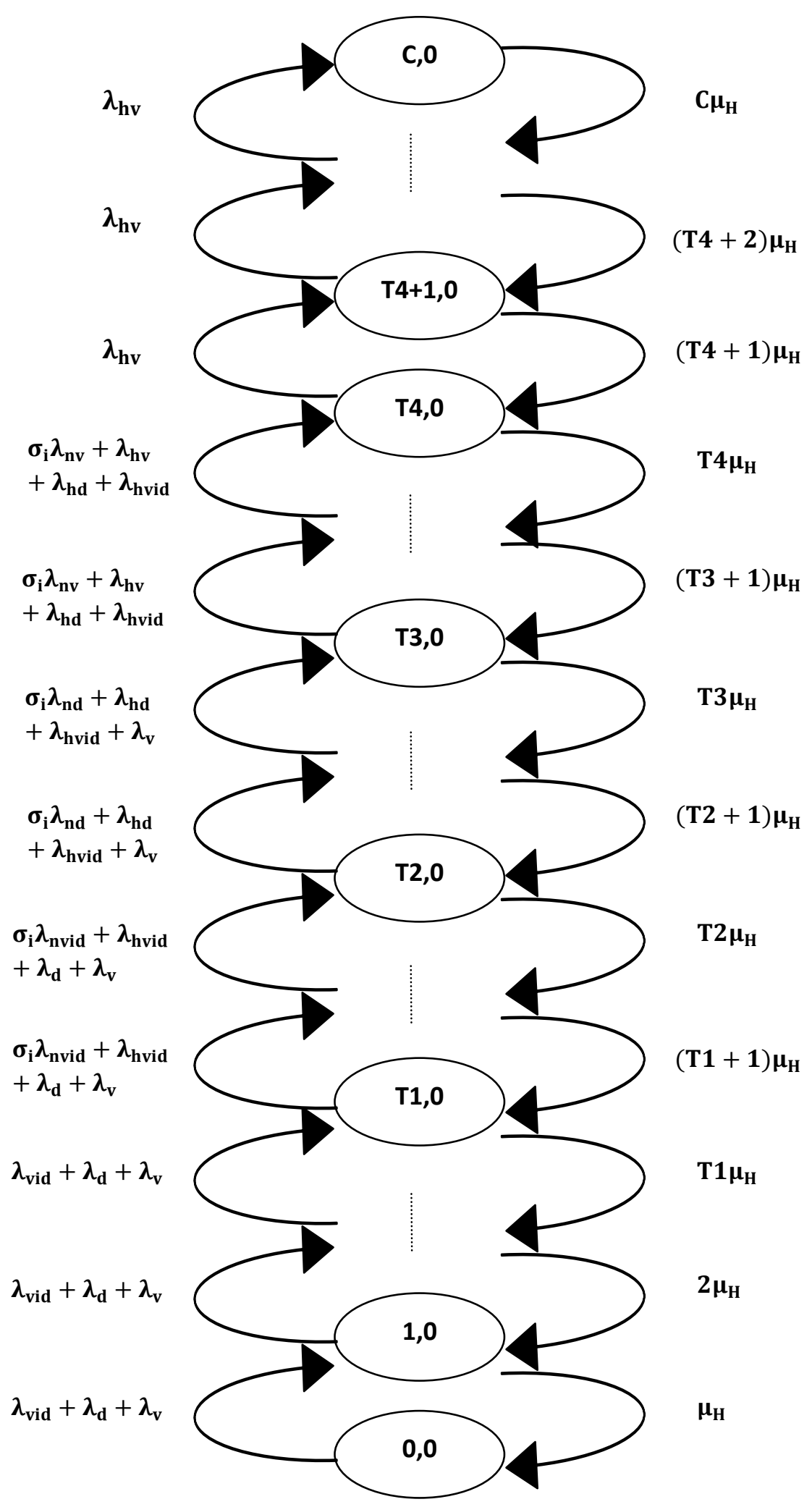

Fig 5: Markov Chain for Main System 


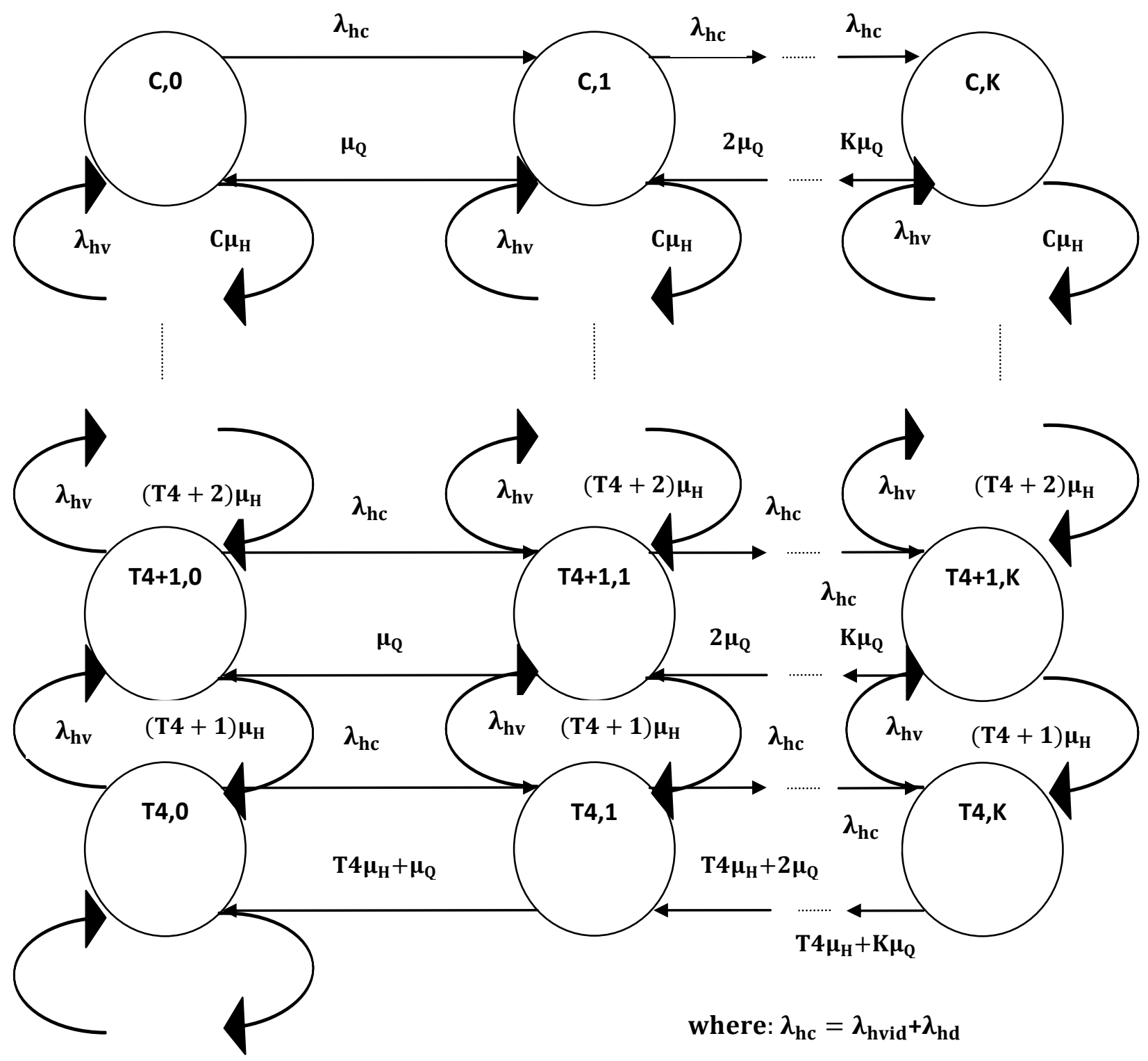

Fig 6: Markov Chain for Queuing System

\section{EVALUATING QOS PERFORMANCE OF THE QDCRS}

In evaluating the QoS of the QDCRS proposed model, the MATLAB software is utilised. The derived equations of (10) are used to help compute the $\mathrm{P}_{\mathrm{B}}, \mathrm{P}_{\mathrm{D}}$, and $\mathrm{U}$ of the system. In pursuing these computations, each individual performance measure of a service is analyzed separately. The objective is to attain a low dropping probability of handoff calls and a low blocking probability of new calls while at the same time achieve efficient system utilization without overload.

To begin, the blocking probability of new video calls $\left(P_{b v i d}\right)$, defined as the sum of the steady state probabilities that requests for new calls are not accepted between $\mathrm{T} 1$ and $\mathrm{C}$, is expressed as the following:

$P_{\text {bvid }}=\sum_{i=T_{1}}^{T_{2}-1}\left(1-\sigma_{i}\right) P(i, 0)+\sum_{i=T_{2}}^{T_{3}-1} P(i, 0)+\sum_{i=T_{3}}^{T_{4}-1} P(i, 0)+$ $i=T 4 C j=O K P(i, j)$
Similarly, the blocking probability for new data calls $\left(P_{b d}\right)$ is expressed as:

$P_{b d}=\sum_{i=T_{2}}^{T_{3}-1}\left(1-\sigma_{i}\right) P(i, 0)+\sum_{i=T_{3}}^{T_{4}-1} P(i, 0)+\sum_{i=T_{4}}^{C} \sum_{j=0}^{K} P(i, j)$

Whereas the blocking probability for new voice calls $\left(P_{b v}\right)$ is given as:

$P_{b v}=\sum_{i=T_{3}}^{T_{4}-1}\left(1-\sigma_{i}\right) P(i, 0)+\sum_{i=T_{4}}^{C} \sum_{j=0}^{K} P(i, j)$

The dropping probability for a handoff call (video and data calls) $\left(P_{d h}\right)$ on the other hand, is defined as the probability of all $\mathrm{K}$ positions of the queue $(\mathrm{Q})$ that are occupied $\left(P_{d h}^{\prime}\right)$ plus the probability of handoff calls that cannot get channels while waiting in Q, identified as the probability of handoff failure $\left(P_{f h}\right) . P_{d h}^{\prime}, P_{f h}$, and $P_{d h}$ are expressed as the following: 


$$
\begin{aligned}
& P_{d h}^{\prime}=\sum_{i=T_{4}}^{C} P(i, K) \\
& P_{f h}=\frac{1}{\left(\lambda_{\text {hd }}+\lambda_{\text {hvid }}\right)} \sum_{i=T_{4}}^{C} \sum_{j=1}^{K} P(i, j) * j * \mu_{Q} \\
& P_{d h}=P_{d h}^{\prime}+P_{f h}
\end{aligned}
$$

The dropping probability of handoff voice calls $\left(P_{d v}\right)$, defined as the probability that all channels are occupied, is determined as:

$$
P_{d v}=\sum_{j=0}^{K} P(C, j)
$$

The system utilisation (U), another performance merit, is defined as the average fraction of active servers in the system expressed as:

$$
\mathrm{U}=\frac{1}{C}\left(\sum_{i=1}^{T_{4}-1} i * P(i, 0)+\sum_{i=T_{4}}^{C} \sum_{j=0}^{K} i * P(i, j)\right)
$$

With expressions (11-18), the performance measures are evaluated and what is sought is the average for each measure to provide an overview of how the QDCRS performs. This performance is compared with the FSS which utilises the following steady state probability:

$$
P(i)=\frac{\left(\lambda_{\text {vid }}+\lambda_{\mathrm{d}}+\lambda_{\mathrm{v}}\right)^{i}}{i !\left(\mu_{\mathrm{H}}\right)^{i}} P(0) \text { for } 0 \leq i \leq C
$$

Where the blocking and dropping probability for all services are:

$$
P_{b}=P_{d}=\sum_{i=0}^{C} P(i)
$$

And the system utilisation is:

$$
\mathrm{U}=\frac{1}{C}\left(\sum_{i=0}^{C} i * P(i)\right)
$$

\subsection{Simulating Results and Discussion}

Table 1 provides the parameters that were used in the MATLAB simulation to validate the analytical model

Table 1. Simulating Parameters

\begin{tabular}{|c|c|}
\hline Mobility & $0,0.4,0.8,1.2,1.6,2.0$ \\
\hline Total \# of Channels & 100 \\
\hline $\begin{array}{c}\text { Threshold Values (T1, T2, } \\
\text { T3, T4) }\end{array}$ & $100 \mathrm{sec}$ \\
\hline $\begin{array}{c}\text { Mean Channel Holding } \\
\text { Time }\left(\frac{1}{\mu_{H}}\right)\end{array}$ & $5 \mathrm{sec}, 90,95$ \\
\hline $\begin{array}{c}\text { Mean Dwell Time }\left(\frac{1}{\mu_{Q}}\right) \\
\text { Queue Size }\end{array}$ & 1 \\
\hline load $(\rho)$ & 0.95 Erlangs \\
\hline
\end{tabular}

The following are the obtained performance results using equations (11-21). The interest was to view the results at the point when all channels were full and to observe whether the QoS was satisfied even at the period when the system was completely occupied.

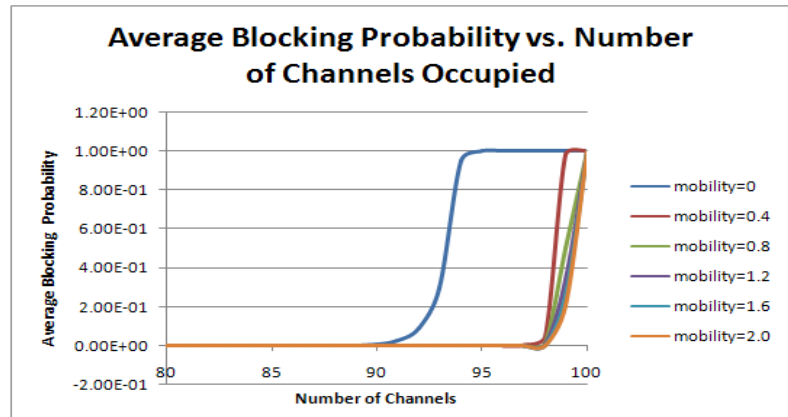

Fig 7: Average Blocking Probability vs. Number of Channels Occupied for DCRS with K=0 (No Queue)

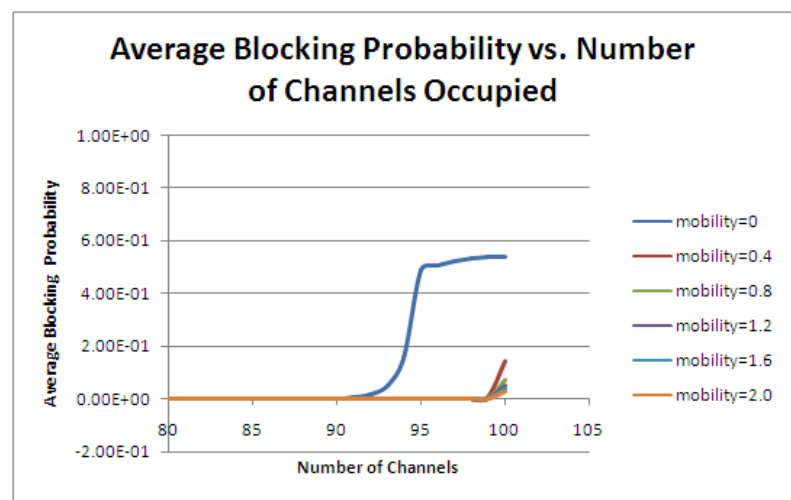

Fig 8: Average Blocking Probability vs. Number of Channels Occupied for DCRS with $\mathrm{K}=1$

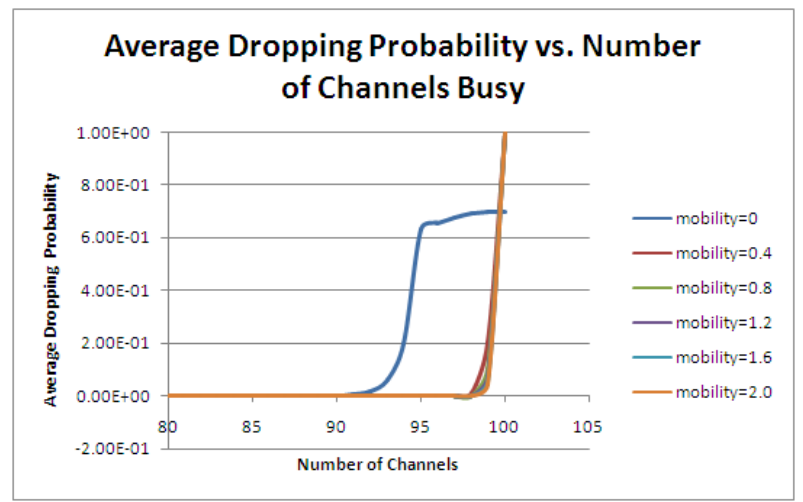

Fig 9: Average Dropping Probability vs. Number of Channels Occupied for DCRS with K=0 (No Queue)

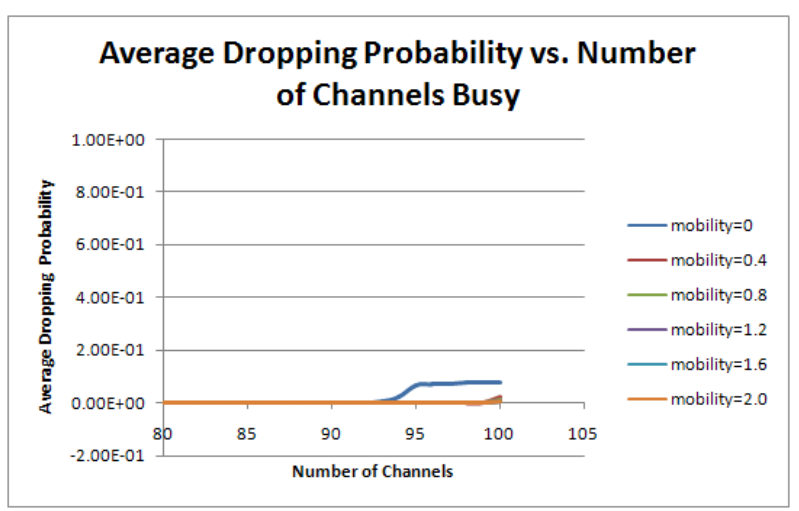

Fig 10: Average Dropping Probability vs. Number of Channels Occupied for DCRS with $K=1$ 
Observing Figures 7 to 10, it can be seen that the DCRS with a queue of size $K=1$ provided desirable results in terms of $P_{B}$ and $P_{D}$ than the DCRS with no queue when approaching a completely occupied system of the 100 allocated channels. This proved the relevance of the Q inclusion into the DCRS system model to improve performance. Furthermore, a $\mathrm{Q}$ with an even bigger capacity $\mathrm{K}$ will attribute an even lower $\mathrm{P}_{\mathrm{B}}$ and $P_{D}$ showing that the QDCRS gives room for an additional performance improvement.

In terms of mobility and considering the definition as the ratio of the mean handoff arrival rate to the mean new arrival rate, it can be seen from the Figures that as mobility increases, meaning the handoff arrival rate is greater than the new arrival rate, $P_{B}$ and $P_{D}$ decreases. This is what is desired and fulfills the objective in ensuring better handoff call performance than new calls.

The performance of QoS for the QDCRS with capacity K=1 and mobility $\alpha=2$ was now compared with the conventional FSS. The following are the results:

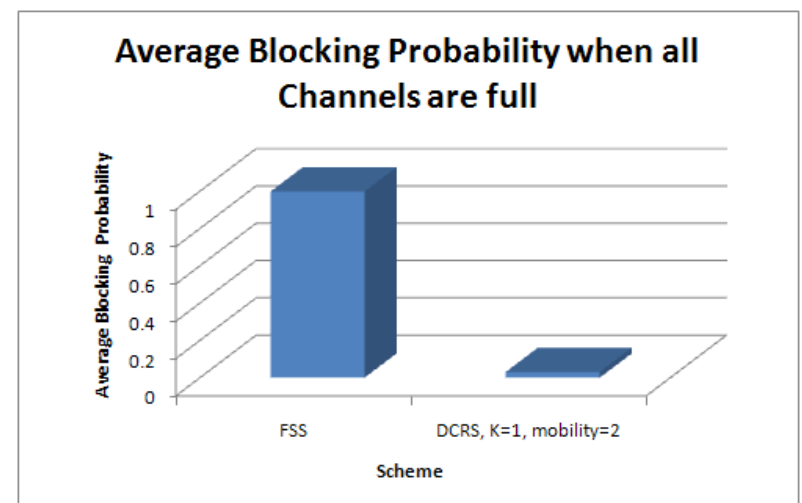

Fig 11: Average Blocking Probability when all Channels are full per scheme

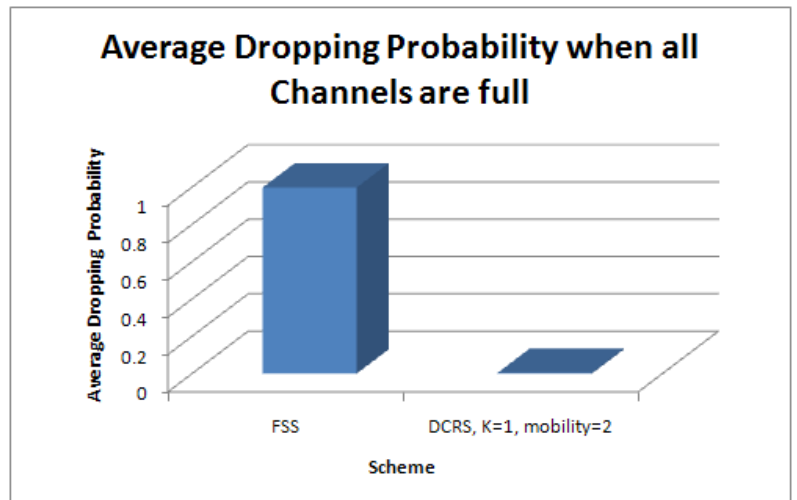

Fig 12: Average Dropping Probability when all Channels are full per scheme

Again it can be seen from Figures 11 to 12, the QDCRS performed much better than the FSS in terms of both $\mathrm{P}_{\mathrm{B}}$ and $\mathrm{P}_{\mathrm{D}}$ attributing much lower probabilities even when all channels are full. In terms of system utilization, as Figure 13 demonstrates, the QDCRS attributes better system utilization when all channels are full compared to the FSS that seems to be overworked when the system is fully in use. Clearly, the benefits of an included Q system are apparent.

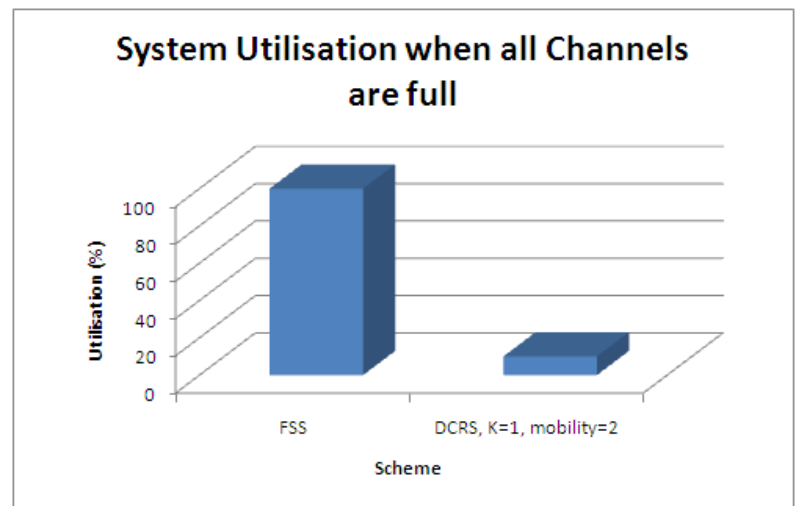

Fig 13: System Utilization when all Channels are full per scheme

Using Little's Theorem formula, the average waiting time $\left(\mathrm{T}_{\mathrm{W}}\right)$ of a handoff call in $\mathrm{Q}$ can be determined by the following equation:

$$
T_{W}=\frac{L_{Q}}{\left(\lambda_{\text {hd }}+\lambda_{\text {hid }}\right)\left(1-\mathrm{P}_{\text {dh }}\right)}
$$

Where $L_{Q}$ is the average queue length given as:

$$
L_{Q}=\sum_{j=1}^{K} j \sum_{i=T_{4}}^{C} P(i, j)
$$

With equations (22) and (23), it was computed that for $\mathrm{K}=1$ and $\alpha=2$, the average wait time for a handoff call in $\mathrm{Q}$ was $0.3 \mu$ s whereas for $\mathrm{K}=2$ and $\alpha=2$, the average wait time in $\mathrm{Q}$ was $0.02 \mathrm{~ns}$. From this, it was clear to see that as $\mathrm{K}$ grew bigger in capacity $\mathrm{T}_{\mathrm{W}}$ decreased justifying that $\mathrm{T}_{\mathrm{W}}$ was inversely proportional to $\mathrm{K}$ which is desired in the performance operation of the QDCRS.

\section{CONCLUSION}

This paper aimed to present a system model that will guarantee the QoS performance of a video, data, and voice integrated wireless cellular network. It was discovered that through the QDCRS model the desired QoS performance of a low $P_{B}$ for new calls, a low $P_{D}$ for handoff calls, and an efficient $U$ where the active servers are not overworked even when all channels are occupied was achieved. Compared to the FSS system and the DCRS, this study showed how relevant the inclusion of a queue system was in ensuring adequate QoS performance and most importantly establishing better performance for handoff calls which is an ideal operation objective for user satisfaction. Furthermore, with an even bigger $\mathrm{Q}$ capacity, wait time for handoff calls can be negligent reducing handoff call failure even more. From this work it can be concluded that the QDCRS with the proposed mobility scheme will ensure adequate QoS for a video, data, and voice integrated cellular network and hence ensure better handoff call performance for the system.

\section{REFERENCES}

[1] Lee E..A., Messerschmitt D.G., "Integrated Networks: In Digital Communication," Springer, Dordrecht, 1988.

[2] A. S. Acampora and M. Naghshineh, "Control and Quality-of Service Provisioning in High Speed Microcellular Networks," IEEE Personal Communications,., 2nd qtr., 1994, pp. 36-43.

[3] N. D. Tripathi, J. H. Reed, and H. F. VanLandingham, "Handoff in Cellular Systems," IEEE Personal Communications, December 1998, pp.26-37. 
[4] S.-C. Kim, "Requirements on WATM Mobile ATM," ATM_Forum/WATM96-1346.

[5] Shun-Fang Yang and Jung-Shyr Wu, "Guard Channel based Call Admission Control Schemes in Hierarchical Mobile IPv6 Networks," International Journal of Communication Networks and Information Security (IJCNIS) Vol. 2, No. 2, August 2010, pp. 68-76.

[6] Jahangir khan, "Handover management in GSM cellular system," International Journal of Computer Applications, Volume 8- No.12, October 2010, pp. 14-24.

[7] A. E. Xhafa and O. K. Tonguz, "Handover Performance of Priority Schemes in Cellular Networks," in IEEE Transactions on Vehicular Technology, vol. 57, no. 1, Jan. 2008, pp. 565-577.

[8] A. A. Adewale, S. N. John, and E. R. Adagunodo, "Performance Comparison of Dynamic Guard Channel Assignment with Buffered Prioritized Scheme for Mobile WiMAX Network," Proceedings of the World Congress on Engineering 2016 Vol I WCE 2016, June 29 - July 1, 2016, London, U.K.

[9] Young Chon Kim, Dong Eun Lee, Bong Ju Lee, Young Sun Kim and B. Mukherjee, "Dynamic channel reservation based on mobility in wireless ATM networks," in IEEE Communications Magazine, vol. 37, no. 11 , Nov 1999, pp. 47-51.

[10] Majid Ghaderi and Raouf Boutaba, "Call Admission Control in Mobile Cellular Networks: A Comprehensive Survey," Wireless Communications \& Mobile Computing, Volume 6 Issue 1, Feb. 2006, pp. 69-93.

[11] Sharif Alagha, "Queuing-Based Dynamic Multi-Guard Channel Scheme for Voice/Data Integrated Cellular Wireless Networks," Masters Thesis, Eastern Mediterranean University, Gazimağusa, North Cyprus, Jan 2013.

[12] M. Aman, S. Mahfooz, and W. U. Rehman, "A Handoff using Guard Channels Scheme (HGCS) for Cognitive Radio Networks," Global Journal of Computer Science and Technology, Vol. 11 Issue 20, Dec.2011.

[13] Muhammed Salamah, "An Adaptive Multi-Guard Channel Scheme for Multi-Class Traffic in Cellular Networks", In proceeding of: IEEE/ACS International 47 Conference on Computer Systems and Applications (AICCSA 2006), Dubai/Sharjah, UAE, 2006.

[14] R. Beraldi, S. Marano and E. Palumbo, "Analysis of new priority queuing strategies for handoff and originating calls in mobile cellular radio systems," IEEE Wireless Communication System Symposium, 1995, pp. 63-69. 